\title{
CARCINOME ANAPLASIQUE DE LA THYROÏDE: A PROPOS DE 11CAS.
}

\section{ANAPLASIC CARCINOMA OF THYROÏD GLAND}

\author{
S. KADI, R. MANI, M. BELCADHI, F. CHARFEDDINE, M. MOKNI*, M. BEN ALI, \\ M. ABDELKÉFI, K. BOUZOUITA. \\ SERVICE D'OTO-RHINO-LARYNGOLOGIE ET DE CHIRURGIE CERVICO-FACIALE. \\ * LABORATOIRE D'ANATOMIE ET DE CYTOLOGIE PATHOLOGIQUE. \\ HÔPITAL UNIVERSITAIRE F. HACHED - SOUSSE.
}

\begin{abstract}
RESUME
Introduction : le carcinome anaplasique de la thyroïde est une tumeur rare, mais agressive. Le but de notre travail est d'étudier les aspects cliniques et de discuter les modalités thérapeutiques de ce cancer.

Matériel et méthodes : Il s'agit d'une étude rétrospective de 11 cas de carcinomes anaplasiques de la thyroïde colligés et traités au service d'ORL et de CCF du CHU Farhat Hached Sousse sur une période de 16 ans (1990-2005).

Résultats : l'âge moyen de nos malades était de 60 ans, il s'agissait de 7 femmes et 4 hommes.

Les signes de compression locale étaient présents dans 7 cas et l'examen physique a objectivé un goitre chez 8 malades avec une taille moyenne de $6 \mathrm{~cm}$. L'atteinte ganglionnaire était observée dans 8 cas et les métastases à distance dans 2 cas.

4 malades ont eu une trachéotomie en urgence avec biopsie thyroïdienne. Les 7 autres malades ont eu une thyroïdectomie totale associée à un curage cervical bilatéral. La radiothérapie a été indiquée dans 10 cas, elle était palliative dans 6 cas. L'évolution était fatale chez 10 malades avec une survie inférieure à un an. Une seule patiente est en vie, en rémission complète avec un recul de 10 ans.

Conclusion : malgré les multiples modalités thérapeutiques envisagées, le pronostic du carcinome anaplasique de la thyroïde reste fâcheux avec une survie ne dépassant qu'exceptionnellement un an.
\end{abstract}

\section{SUMMARY}

Introduction: thyroid anaplasic carcinoma of is a rare, but aggressive tumour. Our aim is to study the clinical aspects and to discuss the therapeutic methods of this cancer with great metastatic capacity and bad prognosis.

Material and methods: It is a retrospective study of 11 cases of thyroid anaplasic carcinoma treated in the ENT department of Farhat Hached Hospital over one 16 years period (1990-2005).

Results: the average age of our patients was 60 years with a female prevalence. The signs of local compression were present in 7 cases and the physical examination objectified a goitre among 8 patients with an average size of $6 \mathrm{~cm}$. Lymph nodes were observed in 8 cases and metastases were noted among 2 patients.

4 patients had a tracheotomy in urgency and 7 other patients had a total thyroïdectomy associated to a bilateral lymph node dissection. The radiotherapy was indicated in 10 cases, palliative in 6 cases. The survival was less than one year for 10 patients. Only one patient is still alive with a complete remission after 10 years of follow up.

Conclusion: in spite of the multiple therapeutic methods considered, the prognosis of anaplasic carcinoma of the thyroid is very bad with a survival exceeding rarely a year.

\section{INTRODUCTION}

Le carcinome anaplasique de la thyroïde, également dénommé carcinome indifférencié est une variété rare de carcinome thyroïdien. II représente 4 à $14 \%$ des tumeurs malignes de la thyroïde [1].

Contrairement aux cancers différenciés, son pronostic est effroyable, la survie ne dépasse qu'exceptionnellement un an.

Les patients sont souvent métastatiques lors du diagnostic mais le décès est le plus souvent lié à une évolution locale de la tumeur.

Le but de ce travail est d'étudier les aspects cliniques et de discuter les difficultés diagnostiques et les modalités thérapeutiques des carcinomes anaplasiques de la thyroïde à partir d'une étude comportant onze malades pris en charge au service d'ORL et de chirurgie cervico-faciale de Sousse.

\section{MATÉRIEL ET MÉTHODES}

Notre travail a consisté en une étude rétrospective de onze observations de malades présentant un carcinome anaplasique de la thyroïde diagnostiqués et traités durant une période de 16 ans allant de 1990 à 2005.

Le diagnostic était toujours vérifié par une relecture des lames histologiques. Dans les cas douteux, une étude immuno-histochimique a été réalisée, utilisant comme 
marqueurs tumoraux la cytokératine et la vimentine.

Nous avons établi pour chaque malade une fiche de renseignements comportant : l'âge, le sexe, les circonstances de découverte, les données cliniques et para cliniques, la prise en charge thérapeutique et les modalités évolutives.

\section{RESULTATS}

Nous avons colligé 11 observations de carcinomes anaplasiques de la thyroïde parmi un nombre total de 185 cancers thyroïdiens traités au cours de la même période ; soit un pourcentage de $6 \%$.

L'âge de nos malades variait de 30 à 76 ans, avec une moyenne de 60 ans. II s'agissait de 4 hommes et 7 femmes.

Le délai de consultation variait de 3 semaines à un an avec une moyenne de 3 mois.

Le motif de consultation était une modification des caractères d'un goitre multi nodulaire ancien chez 7 malades. II s'agissait soit d'une augmentation rapide de son volume soit d'une sensibilité récente en regard de la région basicervicale antérieure. Les 4 autres malades ont consulté pour un nodule thyroïdien découvert par auto palpation ou lors d'un examen systématique.

Dans notre série, 7 patients avaient à l'admission des signes de compression locale représentés par une dysphonie dans 5 cas, une dysphagie haute dans 4 cas et une dyspnée laryngée dans 5 cas. Aucun de nos malades ne décrivait de signes de dysthyroïdie clinique.

L'examen cervical avait objectivé un volumineux goitre dans 8 cas. La taille moyenne de ce goitre était de $6 \mathrm{~cm}$ avec un pôle inférieur non perçu en position d'extension cervicale dans 4 cas. Une fixité par rapport aux plans cutané et musculaire a été notée chez 4 malades, une consistance dure chez 5 malades et une douleur à la palpation chez 2 malades.

L'atteinte ganglionnaire était observée chez 8 patients, elle était de localisation jugulo-carotidienne moyenne et/ou basse. II s'agissait d'adénopathies multiples dont la plus volumineuse mesurait en moyenne $5 \mathrm{~cm}$.

La laryngoscopie indirecte avait objectivé une paralysie laryngée chez 6 malades, celle-ci était bilatérale dans 2 cas.

L'échographie cervicale a été pratiquée chez 10 malades et avait montré un goitre multi nodulaire dans tous les cas. La présence de nodules hétérogènes calcifiés a été notée chez 4 malades. L'échographie a permis aussi de détecter chez deux malades des adénopathies infra cliniques.

La scintigraphie thyroïdienne au technétium 99 pratiquée chez tous nos patients a montré la présence de nodules hypofixants dans 10 cas et une cartographie blanche dans un cas.

Une TDM cervico-thoracique a été réalisée dans 8 cas. Elle a confirmé le caractère plongeant du goitre dans 4 cas. Cet examen a montré une importante déviation de l'axe oeso-trachéal chez 4 malades et la présence d'adénopathies médiastinales chez 2 malades.

Un bilan hormonal thyroïdien comportant le dosage de la T4 et de la TSH pratiqué systématiquement, a révélé une euthyrö̈die biologique chez 10 malades. Une seule patiente était en hyperthyroïdie périphérique.

Parmi les patients qui avaient une dyspnée laryngée associée à une dysphagie haute, deux ont eu une pan endoscopie pré opératoire. Celle-ci était normale dans le premier cas et a révélé une compression extrinsèque de la trachée et de l'œsophage cervical sans atteinte ni effraction de la muqueuse dans le deuxième cas.

4 malades ont eu une trachéotomie en urgence avec biopsie thyroïdienne pour confirmation diagnostique. 7 malades ont eu une thyroïdectomie totale avec un curage médiastino-récurrentiel et cervical bilatéral.

Le diagnostic a été toujours suspecté à l'examen extemporané. L'examen anatomopathologique définitif de la pièce opératoire a confirmé le diagnostic en montrant une prolifération d'un carcinome anaplasique à cellules fusiformes dans 7 cas et à grandes cellules dans 4 cas.

L'examen du reste du parenchyme n'a pas révélé de carcinome différencié associé.

L'effraction capsulaire a été notée chez 8 malades, associée à une infiltration ganglionnaire médiastino-récurrentielle ou cervicale sur les ganglions prélevés.

Un bilan d'extension locorégional et à distance a été pratiqué chez 8 malades, comportant un scanner cervico-thoracique dans 8 cas, une échographie abdominale dans 5 cas et une scintigraphie osseuse dans 2 cas. Deux malades avaient des métastases à distance : un patient avait des métastases pulmonaires multiples et osseuses lombaires basses et un autre avait des métastases pulmonaires.

Une radiothérapie externe a été indiquée chez 10 patients, elle était à visée palliative chez 6 d'entre eux. Une seule patiente avait achevé sa radiothérapie et a reçu une dose totale de 45 Gy avec fractionnement et étalement classique. Les autres malades étaient décédés avant ou au cours de la radiothérapie.

Pour les malades qui ont eu une trachéotomie en urgence avec biopsie thyroïdienne (4 malades), l'évolution immédiate a été émaillée de complications : altération de l'état général, dyspnée aigue, aphagie et amaigrissement important.

Pour les malades qui ont eu initialement une thyroïdectomie totale associée à un évidement cervical bilatéral suivie d'une radiothérapie externe (7 malades), deux patients ont présenté une récidive locale dans un délai de 2 et 8 mois.

L'évolution était fatale pour 10 patients dans un délai de 3 semaines à un an. Une seule patiente est toujours en vie et en rémission complète avec un recul de 10 ans. Le tableau $n^{\circ}$ ㅇ résume les données cliniques, thérapeutiques et évolutives de nos malades. 


\section{OBSERVATIONS PARTICULIÈRES - Première observation :}

II s'agit d'une patiente âgée de 44 ans qui a consulté pour une tuméfaction basi-cervicale antérieure évoluant depuis un an. La patiente ne décrivait pas de signes de compression locale ni de signes de dysthyroïdie.

L'examen a noté la présence d'un nodule thyroïdien lobaire gauche de $4 \mathrm{~cm}$ de grand axe, ferme, indolore, mobile par rapport aux deux plans. II n'y avait pas d'adénopathies cervicales palpables et les cordes vocales étaient mobiles à la laryngoscopie indirecte.

L'échographie cervicale a montré la présence d'un nodule thyroïdien lobaire gauche. Ce nodule était hypofixant à la scintigraphie thyroïdienne.

La patiente a été opérée d'une lobo-isthmectomie gauche. L'examen histologique extemporané était en faveur d'un carcinome anaplasique de la thyroïde. Une totalisation et un curage cervical fonctionnel bilatéral a été alors réalisé. Le diagnostic a été confirmé par l'examen anatomopathologique définitif en montrant une prolifération localisée au niveau du lobe gauche d'un carcinome indifférencié à cellules fusiformes. Le lobe controlatéral était sain. II n'y avait pas d'effraction capsulaire ni d'atteinte ganglionnaire.

Une radiothérapie externe a été entamée 40 jours après l'intervention. La dose délivrée était de $45 \mathrm{~Gy}$, à raison de 2 Gy par séance et avec un étalement sur 6 semaines. L'évolution était favorable sans récidive locorégionale ni métastases à distance avec une survie de 10 ans.

Une relecture des lames avec une étude immuno-histochimique a été demandée à deux reprises et a confirmé le diagnostic du carcinome anaplasique de la thyroïde. La tumeur était localisée au niveau du lobe gauche sans effraction capsulaire ni extension locorégionale.

\section{Deuxième observation :}

II s'agissait d'une patiente âgée de 30 ans, qui a consulté pour une tuméfaction basicervicale antérieure évoluant depuis 10 jours et augmentant rapidement de volume.

A l'examen, elle avait un goitre ferme, hétérogène et douloureux à la palpation avec des signes inflammatoires locaux, associé à des adénopathies cervicales jugulocarotidiennes bilatérales, sensibles et mobiles par rapport aux deux plans.

Le bilan biologique a montré une vitesse de sédimentation à $83 \mathrm{~mm}$ à la première heure et une fibrinémie à $6 \mathrm{~g} / \mathrm{l}$. Le dosage des hormones thyroïdiennes a révélé une hyperthyroïdie périphérique.

L'échographie cervicale a objectivé l'existence d'un goitre hétérogène associé à de multiples adénopathies d'allure inflammatoire.

Ce goitre n'avait pas de fixation à la scintigraphie thyroïdienne.

Le diagnostic d'une thyroïdite de De Quervain a été fortement suspecté, et un traitement médical à base de corticoïdes par voie générale a été instauré.
L'évolution était défavorable avec apparition, à $\mathrm{J} 10 \mathrm{du}$ traitement, d'une diplégie laryngée associée à une dyspnée aigüe. Une trachéotomie en urgence a été pratiquée avec une biopsie thyroïdienne dont le résultat a conclu à un carcinome anaplasique de la thyroïde.

L'évolution était fatale en moins d'un mois.

\begin{tabular}{|c|c|c|c|c|c|c|c|}
\hline Age & Sexe & $\begin{array}{l}\text { Signes de } \\
\text { compression }\end{array}$ & $\begin{array}{l}\text { Talle } \\
\text { Tumorale } \\
(\mathrm{cm})\end{array}$ & $\begin{array}{l}\text { ADP } \\
(\mathrm{cm})\end{array}$ & M & Traitement & $\begin{array}{l}\text { Survie } \\
\text { (mois) }\end{array}$ \\
\hline 60 & $\mathrm{~F}$ & + & 6 & 5 & - & $\mathrm{TT}+$ curage+ $\mathrm{RT}$ & 3 \\
\hline 30 & $\mathrm{~F}$ & + & 4 & 2 & - & Trachéotomie+biopsie & $<1$ \\
\hline 53 & M & + & 5 & 3 & - & $\mathrm{TT}+$ curage $+\mathrm{RT}$ & 1 \\
\hline 44 & $\mathrm{~F}$ & - & 4 & - & - & $\mathrm{TT}+$ curage+ $\mathrm{RT}$ & 120 \\
\hline 69 & M & + & 6 & 2 & + & Trachéotomie+Biopsie & $<1$ \\
\hline 74 & $\mathrm{~F}$ & - & 6 & - & - & Trachéotomie+Biopsie & 3 \\
\hline 50 & $\mathrm{~F}$ & - & 8 & 4 & - & $\mathrm{TT}+$ curage+ $\mathrm{RT}$ & 11 \\
\hline 70 & $\mathrm{~F}$ & - & 10 & - & - & $\mathrm{TT}+$ curage+ $\mathrm{RT}$ & 1 \\
\hline 76 & $\mathrm{M}$ & + & 6 & 5 & + & Trachéotomie+RT & 2 \\
\hline 51 & $\mathrm{~F}$ & + & 6 & 3 & - & TT + curage & 1 \\
\hline 72 & $\mathrm{M}$ & + & 8 & 3 & - & $\mathrm{TT}+$ curage+ $\mathrm{RT}$ & 2 \\
\hline
\end{tabular}

Tableau I : Carcinome anaplasique de la thyroïde : aspects cliniques, prise en charge thérapeutique et survie.

\section{DISCUSSION}

Le carcinome anaplasique de la thyroïde se caractérise par une évolution rapide, brutale et par un pouvoir métastatique explosif $[1,2]$.

C'est une tumeur rare. Elle représentant $6 \%$ de l'ensemble des cancers thyroïdiens traités dans notre service durant la période de l'étude.

Dans moins de $10 \%$ des cas, il s'agit d'une transformation d'un carcinome différencié de la thyroïde par mutation du gène P53 [1]. Cette mutation a été retrouvée dans 70 à $85 \%$ des carcinomes indifférenciés de la thyroïde versus 0 à $9 \%$ des carcinomes différenciés. Elle est incriminée dans le changement du caractère moléculaire de la cellule thyroïdienne expliquant sa grande agressivité lors de la transformation anaplasique [3].

Le carcinome anaplasique de thyroïde est exceptionnel avant l'âge de 40 ans, il survient essentiellement chez les sujets âgés avec un pic de fréquence de 60 à 70 ans. La prédominance féminine est rapportée dans toutes les séries $[4,5,6]$.

Dans notre série, l'âge moyen était de 60 ans, avec 7 femmes pour 4 hommes.

Le carcinome anaplasique de la thyroïde se révèle généralement par un volumineux goitre ou un nodule ancien rapidement évolutif $[1,7,8]$.

Les signes d'envahissement loco-régional sont clas- 
siques avec une dyspnée aigue, une dysphonie par paralysie récurrentielle, une dysphagie, un syndrome cave supérieur ou un syndrome de Claude Bernard Horner par compression du plexus nerveux sympathique [9].

Les signes généraux avec altération de l'état général sont fréquents dans le carcinome anaplasique de la thyroïde, observés dans 40 à $50 \%$ des cas [1].

Dans notre série, 8 malades avaient des signes d'envahissement locorégional (dyspnée, dysphonie, dysphagie, cervicalgie) associés dans 4 cas à des signes généraux (amaigrissement et altération de l'état général).

A l'examen, la tumeur est d'allure tout à fait suspecte devant l'évolution rapide, la taille généralement supérieure à $6 \mathrm{~cm}$, la consistance dure, la fixité aux plans profonds et la présence d'adénopathies latérocervicales souvent confondues avec la tumeur $[10,11]$.

Dans notre série, 8 malades avaient tous ces critères cliniques évocateurs de la malignité.

Souvent l'importance du volume tumoral et les troubles compressifs qu'il entraîne imposent, avant tout examen, la pratique d'une trachéotomie de sauvetage avec biopsie thyroïdienne confirmant la nature anaplasique de la tumeur. Selon l'étude de Voutilainen [4], portant sur 33 malades présentant un carcinome anaplasique de la thyroïde, $30 \%$ ont nécessité une trachéotomie en urgence. L'échographie cervicale peut évoquer la malignité si le nodule est hypoéchogène, hétérogène, mal limité avec des contours irréguliers. En plus elle décèle les adénopathies infra cliniques et apprécie l'envahissement local de la tumeur [2]. Dans notre série l'échographie cervicale a été pratiquée chez 9 malades et a évoqué la malignité dans 4 cas en montrant des nodules hétérogène comportant des microcalcifications.

La TDM cervico-thoracique explore mieux l'extension aux structures tissulaires avoisinantes (trachée, œsophage, axes vasculaires et muscles). Son apport est important en cas de goitre plongeant en précisant les limites et les rapports du goitre avec les structures médiastinales [2,8]. Dans le carcinome anaplasique de la thyroïde, la tumeur parait iso-dense ou légèrement hyperdense dans la majorité des cas [12].

La scintigraphie au technétium 99 montre le plus souvent des nodules hypofixants. La scintigraphie a été pratiquée chez tous nos malades, elle a montré des nodules froids dans 10 cas et une cartographie blanche dans un cas.

Le bilan hormonal thyroïdien est en faveur d'une euthyroïdie dans $98 \%$ des cas [2]. Une seule patiente de notre série avait une hyperthyroïdie biologique. Chez cette patiente nous avons suspecté le diagnostic d'une thyroïdite de De Quervain devant I'histoire clinique, le syndrome inflammatoire biologique et la scintigraphie qui était blanche.

L'échographie cervicale et la TDM permettent de guider la cytoponction lorsque le nodule thyroïdien suspect est petit ou profond. Selon Besic, le diagnostic du carcinome ana- plasique de la thyroïde a été posé à partir de l'examen cytologique chez 118 malades au cours d'une étude comportant 162 patients [6].

Cet examen n'est pas de pratique courante dans notre conduite diagnostique. II nécessite en plus d'une technique rigoureuse, un cytologiste bien expérimenté.

Le diagnostic cytologique et même histologique du carcinome anaplasique de la thyroïde n'est pas toujours aisé posant ainsi plusieurs diagnostics différentiels tel que les thyroïdites, le carcinome insulaire, le carcinome médullaire, les lymphomes thyroïdiens et les métastases thyroïdiennes d'un autre carcinome [9,13,14]. D'où l'importance de l'étude immuno-histochimique dans le diagnostic de cette tumeur qui permet de détecter la présence des protéines FAS et de cytokératine et la négativité des autres marqueurs tumoraux classiques des cancers thyroïdiens (thyroglobuline, calcitonine, ACE) [15].

D'autre part, l'étude histologique du reste du parenchyme est nécessaire à la recherche d'un autre carcinome coexistant avec le carcinome anaplasique. En effet, Selon Pierie, cette association a été retrouvée chez $16 \%$ des malades de sa série [11]. Pour Mclver, la coexistence d'un carcinome différenciés de la thyroïde était notée dans $18 \%$ des cas, dont $68 \%$ étaient un carcinome papillaire [16]. Dans notre série, l'examen histologique n'a pas révélé de carcinome différentié associé au carcinome anaplasique de la thyroïde.

Les métastases à distance sont observées dans 20 à $50 \%$ des cas. La localisation plus fréquente est pulmonaire ensuite cérébrale puis osseuse [3]. Dans notre série, le bilan d'extension a révélé des métastases pulmonaires et osseuses chez un homme de 69 ans et des métastases pulmonaires chez un autre homme de 76 ans. Ces deux malades n'avaient pas eu de chirurgie radicale, le deuxième a eu une irradiation à visée palliative.

Le traitement du carcinome anaplasique de la thyroïde repose sur la chirurgie, la radiothérapie et la chimiothérapie. L'association de ces 3 modalités thérapeutiques semble la plus efficace pour améliorer le contrôle local $[3,6,16]$.

Plusieurs auteurs recommandent, dans la mesure de possibilités locales et générales, une thyroïdectomie totale associée à un évidement ganglionnaire cervical bilatéral suivi d'une radiothérapie cervico-médiastinale de 45 à 64 Gy à raison de 2 Gy par séance ; 5 séances par semaine associée ou non à une chimiothérapie : soit à base d'Adriamycine en monothérapie ou de Doxorubicine et Cisplatine en poly thérapie $[4,8,11,12]$.

Selon Kim, une meilleure réponse locale est observée en cas d'utilisation d'une poly chimiothérapie qu'en mono chimiothérapie. Cette réponse est encore améliorée en cas d'association d'une radiothérapie hyper fractionnée [14]. D'autre part, certains auteurs mentionnent que l'indication de la chimiothérapie est limitée par sa toxicité, surtout chez les sujets âgés ou multi tarés [3,2,9]. 
Un essai du traitement par radio-chimiothérapie première suivie d'une exérèse chirurgicale est envisageable actuellement afin de mieux contrôler localement la tumeur $[12,17,18]$. En effet, selon Besic [6], ce protocole de chimio-radiothérapie première ne semble pas influencer la survie, mais son but est de potentialiser l'effet tumoricide de cette association sur une tumeur connue résistante.

Pierie et Malver ont montré que l'exérèse tumorale radicale curative versus partielle décompressive ne modifie pas le risque de récidive locale ni la survie, et que la radiothérapie post-opératoire ne diminue pas le risque de récidive mais pourrait par contre la retarder $[11,16]$.

Le tableau II résume les résultats thérapeutiques de certaines grandes séries de carcinomes anaplasiques retrouvées dans la littérature $[6,7,8,16]$.

Dans notre étude, 7 malades ont eu une thyroïdectomie totale associée à un curage cervical bilatéral et 4 autres ont eu une trachéotomie de sauvetage associée à une thyroïdectomie de réduction. On n'a pas constaté une meilleure survie chez les malades ayant eu un traitement chirurgical radical, puisque 5 d'entre eux étaient décédés en moins de 3 mois.

Souvent, l'importance et la rapidité de l'extension locorégionale ainsi que le pouvoir métastatique important de cette tumeur, rend la chirurgie radicale impossible se limitant à une simple biopsie ou à une chirurgie palliative de réduction $[2,4]$.

La surveillance est clinique et radiologique rythmée par la symptomatologie ou périodique tous les 3 mois. Son but est d'éviter ou d'alléger la morbidité ainsi que de dépister les récidives locorégionales et les métastases à distance et de rechercher d'éventuelles complications de la radiothérapie $[10,11,12]$.

Plusieurs facteurs conditionnent le pronostic du carcinome anaplasique de la thyroïde - qui reste tout de même très fâcheux - notamment l'âge avancé, la taille de la tumeur, l'effraction capsulaire, la nécrose tumorale, le grand index mitotique, l'envahissement ganglionnaire et les métastases à distance qui sont de très mauvais pronostic $[2,11]$.

La survie à 5 ans est inférieure à $10 \%$, la moyenne de survie est de 6 à 8 mois quelque soit le traitement. S'il existe des métastases, la survie moyenne chute à 3 mois $[8,10,16]$.

Dans notre série, une seule patiente avait une survie de 10 ans. Cette patiente a eu une thyroïdectomie totale suivie d'une radiothérapie. II s'agissait d'une tumeur localisée uniquement au niveau d'un lobe thyroïdien, sans effraction capsulaire ni extension locorégionale ou métastases à distance.

Dans l'étude de Mclver, comportant 134 malades, une seule patiente avait une survie de 23 ans. Cette patiente a eu une thyroïdectomie totale sans radio-chimiothérapie post opératoire [16].

Une survie supérieure à 5 ans a été rapportée par
Goutsouliak chez 2 malades, dans une série comportant 75 cas de carcinomes anaplasiques de la thyroïde. Ces malades ont eu une thyroïdectomie subtotale suivie d'une radiothérapie de 40 à 45 Gy [7].

\begin{tabular}{|c|c|c|c|}
\hline Auteurs & $\begin{array}{l}\text { Nombre de } \\
\text { cas }\end{array}$ & Traitement & Résultats \\
\hline $\begin{array}{c}\text { BESIC } \\
\text { [6] }\end{array}$ & 79 & $\begin{array}{l}\text { (1) Chirurgie première: } \\
\pm \text { RT et/ou CT } \\
\text { (2) RT et/ou CT première : } 53 \\
\pm \text { Chirurgie }\end{array}$ & $\begin{array}{l}\text {-Pas de différence } \\
\text { significative pour l a } \\
\text { survie. } \\
\text {-Amélioration du } \\
\text { contrôle local pour le } \\
\text { groupe (2). }\end{array}$ \\
\hline $\begin{array}{c}\text { GOUTS ULIAK } \\
\text { [7] }\end{array}$ & 75 & $\begin{array}{l}\text { (1) Chirurgie : } 13 \\
\text { * complète: } 4 \\
\text { * Partielle : } 9 \\
\text { (2) Radiothérapie: } 48 \\
\text { * curative : } 15 \\
\text { * palliative : } 33 \\
\text { (3) Chimiothérapie : } 7\end{array}$ & $\begin{array}{c}\text { Meilleure survie: en cas } \\
\text { de chirurgie complète } \\
\text { puis radiothérapie à } \\
\text { dose > à } 40 \mathrm{~Gy} .\end{array}$ \\
\hline $\begin{array}{c}\text { BITTMANN } \\
{[8]}\end{array}$ & 30 & $\begin{array}{l}\text { (1) Chirurgie première : } 20 \\
\text { * complète }: 10 \\
\text { * partielle : } 10 \\
\text { (2) RT/ CT première : } 4\end{array}$ & $\begin{array}{l}\text { Moyenne de survie : } \\
\text { Groupe (1): } 22 \text { mois. } \\
\text { Groupe (2): } 16 \text { mois. }\end{array}$ \\
\hline $\begin{array}{c}\text { MCLVER } \\
\text { [16] }\end{array}$ & 134 & $\begin{array}{l}\text { (1) Chirurgie : } 96 \\
* \text { complète: } 29 \\
\text { * partielle : } 67 \\
\text { (2) Radiothérapie : } 108 \\
\text { * curative : } 79 \\
\text { * palliative : } 29 \\
\text { (3) Chimiothérapie : } 16\end{array}$ & $\begin{array}{l}\text { Chirurgie complète } \\
\text { versus partielle : NS } \\
\\
\text { Radio thérapie curative } \\
\text { versus palliative : NS }\end{array}$ \\
\hline Notre série & 11 & $\begin{array}{l}\text { (1) Chirurgie complète : } 7 \\
\text { (2) Radiothérapie : } 10 \\
\text { * curative }: 4 \\
\text { * palliative : } 6\end{array}$ & $\begin{array}{l}\text { Survie } \leq 3 \text { mois : } 9 \\
\text { Survie }=11 \text { mois : } 1 \\
\text { Survie }=10 \text { ans : } 1\end{array}$ \\
\hline
\end{tabular}

CT : Chimiothérapie - RT : Radiothérapie

Tableau II : Carcinome anaplasique de la thyroïde : Prise en charge thérapeutique et résultats.

\section{CONCLUSION}

Le carcinome anaplasique de thyroïde est une forme agressive et léthale des cancers thyroïdiens.

Les métastases à distance sont précoces, observées dans 20 à $50 \%$ des cas au moment du diagnostic.

Les protocoles thérapeutiques utilisés peuvent être efficaces chez quelques malades. La toxicité aigue est le principal facteur limitant du traitement chez des malades le plus souvent âgés et fréquemment en mauvais état général.

Dans toutes les séries, aucune réponse n'a pu être obtenue sur les métastases à distance. Ceci souligne la nécessité de traiter ces patients le plus rapidement possible, avant l'apparition de métastases.

Par ailleurs, même en présence de métastases à distance, I'association des différentes modalités thérapeutiques disponibles peut être utile parce qu'elle améliore la qualité de vie de ces patients et peut prévenir le décès par asphyxie lié à la progression locale de la maladie. 


\section{REFERENCES}

1. Schlumberger MJ. Anaplastic thyroid carcinoma. Orphanet encyclopedia. 2005:1-4

2. Lakhloufi A, Moulay Rachid M, Khaiz D, Rifki-Jai S, Chehab F, Bouzidi A. Le cancer anaplasique de la thyroïde. Médecine du Maghreb. 1998;70:11-7. 3. Ohtsuru A, Podtcheko AP, Tsuda S and et coll. A novel radiosensitizing therapy for anaplastic thyroid cancers. International Congress Series. 2003;1258:273-

4. Voutilainen PE, Multanen M, Haapianinen RK, Leppaniemi AK, Sivula AH. Anaplastic thyroid carcinoma survival. World J Surg. 1999;23:975-9.

5. Rodriguez JM, Pinero A, Ortiz S et coll. Clinical and histological differences in anaplastic thyroid carcinoma. Eur J Surgery. 2000;166:34-3.

6 . Besic N, Auersperg M, Us-Krasovec et coll. Effect of primary treatment on survival anaplastic thyroid carcinoma. Eur J Surg Oncol. 2001;27:260-4

7. Goutsouliac V, Hay JH. Anaplastic thyroid cancer in British Columbia 19851999: A population-based study. Clin Oncol. 2005;17:75-8.

8. Bittmann O, Bruneton JN, Fenart D et coll. Imaging of anaplastic carcinoma of the thyroid gland. J Radiol. 1992;73:35-8.

9. Sugito K, Ito K, Mimura T et coll. The important role of operations in the management of anaplastic thyroid carcinoma. Surgery. 2002;131:245-8.

10. Lifante JC, Mancini F, Mithieux F et coll. Résultats des résections trachéales ou oesophagiennes dans les carcinomes invasifs de la thyroïde. Ann chirur.
2001;126:236-41.

11. Pierie JP. The effect of surgery and radiotherapy on outcome of anaplastic thyroid carcinoma. Ann surg oncol. 2002;9:57-64.

12. Marc D, Posner M, Jeanne M et coll. Dose optimization for the treatment of anaplastic thyroid carcinoma: a comparison of treatment planning techniques. Int J Radiat Oncol. 2000;48:475-83.

13. Lam K, Lo C, Chan K W. Insular and anaplastic carcinoma of the thyroid: a 45-year comparative study at a single institution and a review of the thyroid significance of p53 and p21. Ann Surg. 2000;231:329-38.

14. Kim JH, Leeper RD. Treatment ob locally advanced thyroid carcinoma with combination Doxorubicin and radiation therapy. Cancer. 1987;60:2372-5.

15. Sckiguchi M, Shiroko Y, Arai T et coll. Biological charateristics and chemosensitivity profile of four humain anaplastic thyroid carcinoma cell lines. Biomed Pharmacother. 2001;55:466-74.

16. Mclver B, Hay ID, Giuffrida DF. Anaplasic thyroid carcinoma: A50-year experience at single institution. Surgery. 2001;130:1028-34

17. Lo C, Lam K, Wan KY et coll. Anaplastic carcinoma of the thyroid. Surgery. 1999; 177: 337-9.

18. Tennvall J, Lundell, Wahlberg P. Anaplastic thyroid carcinoma: Three protocols combining Doxorubicin, hyperfractionated radiotherapy and surgery. $\mathrm{Br}$ Cancer. 2002;86:1848-53. 